\title{
Negative electronic compressibility and nanoscale inhomogeneity in ionic-liquid gated two-dimensional superconductors
}

\author{
G. Dezi, ${ }^{1}$ N. Scopigno, ${ }^{1,2}$ S. Caprara, ${ }^{1,3}$ and M. Grilli ${ }^{1,3, *}$ \\ ${ }^{1}$ Dipartimento di Fisica, Università di Roma Sapienza, P.le Aldo Moro 5, 00185 Roma, Italy \\ ${ }^{2}$ Institute for Theoretical Physics, 3584 CC Utrecht, the Netherlands \\ ${ }^{3}$ ISC-CNR Unità di Roma Sapienza, Roma, Italy
}

(Received 9 May 2018; published 10 December 2018)

\begin{abstract}
When the electron density of highly crystalline thin films is tuned by chemical doping or ionic-liquid gating, interesting effects appear including unconventional superconductivity, sizable spin-orbit coupling, competition with charge-density waves, and a debated low-temperature metallic state that seems to avoid the superconducting or insulating fate of standard two-dimensional electron systems. Some experiments also find a marked tendency to a negative electronic compressibility. We suggest that this indicates an inclination for electronic phase separation resulting in a nanoscopic inhomogeneity. Although the mild modulation of the inhomogeneous landscape is compatible with a high electron mobility in the metallic state, this intrinsically inhomogeneous character is highlighted by the peculiar behavior of the metal-to-superconductor transition. Modeling the system with superconducting puddles embedded in a metallic matrix, we fit the peculiar resistance vs temperature curves of systems like $\mathrm{TiSe}_{2}, \mathrm{MoS}_{2}$, and $\mathrm{ZrNCl}$. In this framework also the low-temperature debated metallic state finds a natural explanation in terms of the pristine metallic background embedding nonpercolating superconducting clusters. An intrinsically inhomogeneous character naturally raises the question of the formation mechanism(s). We propose a mechanism based on the interplay between electrons and the charges of the gating ionic liquid.
\end{abstract}

DOI: 10.1103/PhysRevB.98.214507

\section{INTRODUCTION}

In the last years, great advances have been achieved in the fabrication of idealized two-dimensional (2D) electron systems such as heterogeneous interfaces, molecular-beamepitaxy grown atomic layers, exfoliated thin flakes, and fieldeffect devices. Moreover, the possibility of combining or singling out isolated layers of graphene, transition metal dichalcogenides, high-temperature superconducting cuprates, transition metal oxide interfaces, and so on, has opened new perspectives of a "Lego" functionalization of 2D systems (see, e.g., Ref. [1]). One common relevant feature of these systems is their highly crystalline character, which renders them an ideal playground for studying several intriguing physical effects [2], like, e.g., Ising superconductivity or topological phases, without the complications due to impurity or defect-induced disorder. Among these properties there is the appearance of a low-temperature quantum metallic state, which has been proposed as a new state of matter escaping the standard dichotomy of 2D electron systems, which at low temperature are usually forced to choose between an insulating (typically due to Anderson localization) or a superconducting state. These highly crystalline 2D systems, like transition metal dichalcogenides or $\mathrm{ZrNCl}$ ultrathin films, instead, display a low-temperature resistance saturation. They start from a metallic high-temperature state, then display a suppression of resistance due to the occurrence of

\footnotetext{
${ }^{*}$ Correspondence and requests for materials should be addressed to marco.grilli@roma1.infn.it
}

superconductivity, when the temperature $T$ is lowered. However, when superconductivity is (partially) destroyed by a magnetic field, they display a low- $T$ metallic behavior, often marked by a rather extended plateau, with the resistance extrapolating to a finite constant value. There are some features of the resistance curves $R(T)$ that are quite specific of this anomalous state. First of all, the transition from high- $T$ metal to superconductor (or to low- $T$ metal) is very broad, so broad that by no means its width can be reproduced by standard superconducting fluctuation mechanisms in the manner of Aslamazov-Larkin or Halperin-Nelson. Second, the shape of $R(T)$ is peculiarly "tailish" with a long foot in its low- $T$ side. Traditionally, this behavior is attributed to vortex dissipation [3] but, remarkably, the very same features occur in the absence of magnetic field, when superconductivity is weakened or partially suppressed by reducing the electron density. This indicates that the usual mechanism of vortex dissipation cannot be invoked. The case of $\mathrm{ZrNCl}$ reported in Fig. 1(a), where these features $[R(T)$ saturation and tailish behavior] are highlighted by a gray shadowing, is particularly eloquent.

A possible clue to interpret these unusual features comes from the comparison with the $2 \mathrm{D}$ electron gas formed at the oxide interfaces like, e.g., $\mathrm{LaAlO}_{3} / \mathrm{SrTiO}_{3}$, where the temperature dependence of the resistance curves displays a very similar behavior. Despite their highly crystalline structure, the above peculiar features have been successfully explained in these systems in terms of nanoscale electronic inhomogeneities [4-7]. Further indications that oxide interfaces and other 2D electron systems are peculiarly inhomogeneous come from the observation of a quantum Griffiths state when a 

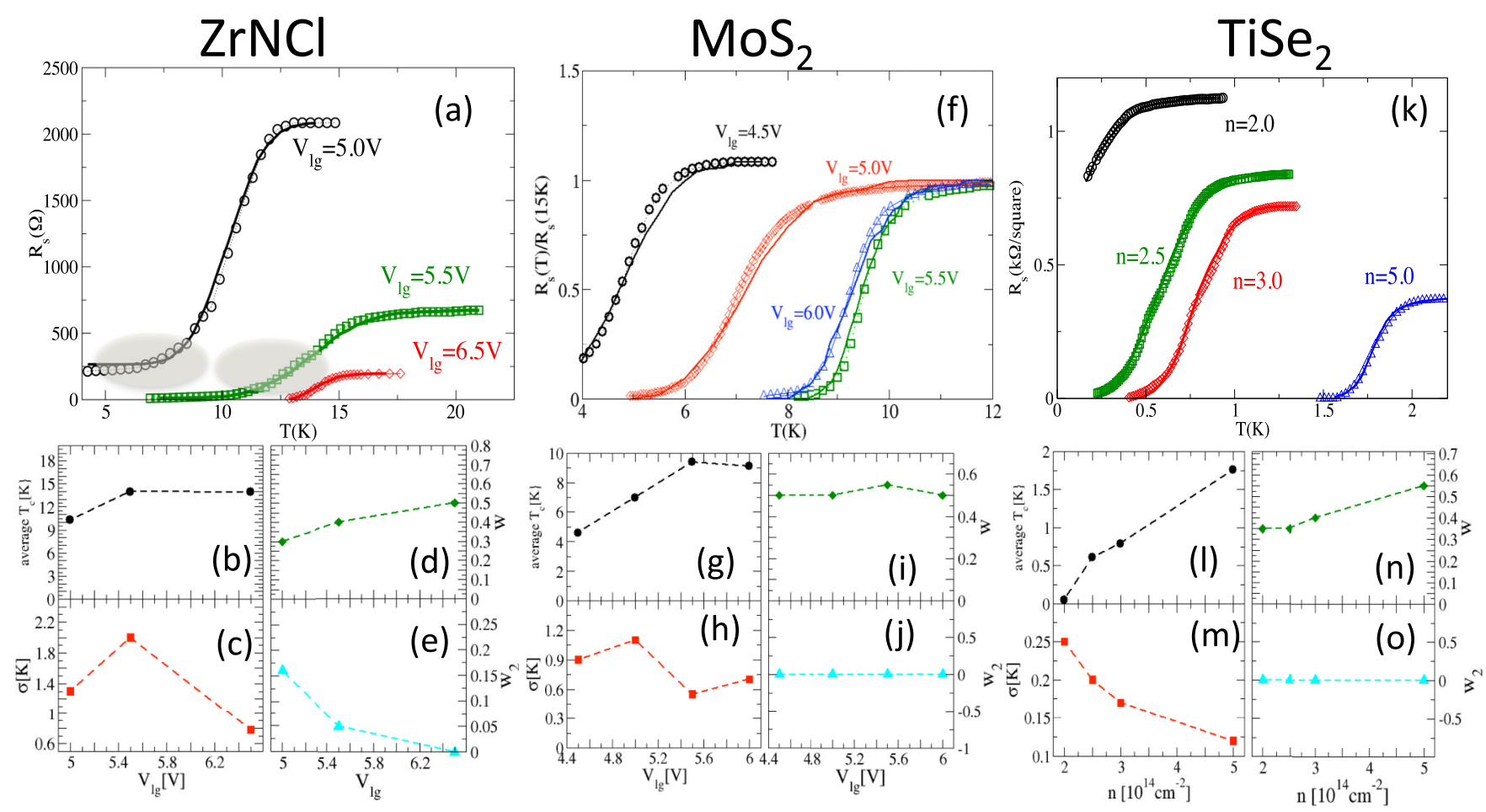

FIG. 1. Fit (symbols) and experimental resistance (solid curves) (a) of $\mathrm{ZrNCl}$ (from Ref. [15]) at three different values of the ionic-liquid gating $\left(V_{l g}=5.0,5.5\right.$, and $\left.6.5 \mathrm{~V}\right)$. (b)-(e) The average local $\bar{T}_{c}$, the variance $\sigma$ of their distribution, the total weight $w$ of the superconducting regions, and the fraction $w_{2}$ of broken bonds in the filamentary superconducting cluster (see Appendix) as resulting from the fits. (f)-(j) Same as in (a)-(e) for the $\mathrm{MoS}_{2}$ experiments of Ref. [14] ( $V_{l g}=4.5,5.0,5.5$, and 6.0 V). (k)-(o) Same as (a)-(e) and (f)-(j) for the TiSe 2 experiments of Ref. [16] (electron density $n=2.0,2.5,3.0$, and $5.0 \times 10^{14} \mathrm{~cm}^{-2}$ ). The electron densities reported in the legend of $(\mathrm{k})$ are in the same $10^{14}$ electrons per $\mathrm{cm}^{-2}$ units of panels (1)-(o). The shaded regions in (a) highlight the tailish character of the resistance curves and the saturating plateau at low electron density and temperature, marking the regime without a percolating superconducting subset.

perpendicular magnetic field drives a metal-to-superconductor transition $[8,9]$. Of course, inhomogeneity might seem at odds with the remarkably ordered structure of these systems and the high mobility of the charge carriers indicates that the usual low- $T$ scattering mechanisms (crystal defects and impurities) are not of primary relevance in these systems. This suggests that inhomogeneities are not induced by extrinsic sources, but likely occur from intrinsic mechanisms [10,11] that destabilize the electronic liquid giving rise to (short-scale) electronic phase separation and density fluctuations on the nanoscopic scale.

In this work we consider transport experiments and, from the analysis of resistivity curves near the metal-tosuperconductor transition, we provide a clear evidence of the inhomogeneous character of some $2 \mathrm{D}$ highly crystalline systems like transition metal dichalcogenides and $\mathrm{ZrNCl}$, and we deduce the structure of this inhomogeneity. We focus on the metal-to-superconductor transition driven by changing the electron density in the absence of magnetic field, so that the observed transport properties should be interpreted without invoking the dissipation effects of vortices. The model we adopt, although based on reasonable assumptions, is phenomenological in nature and does not aim at identifying the microscopic mechanisms underlying the inhomogeneity formation, its structural and electronic properties, and so on. It rather aims at introducing the minimal amount of knobs to be tuned in order to reproduce the data and to extract information on the inhomogeneity (the fraction of high- vs low-density regions, the connectivity of the inhomogeneous clusters, the way superconductivity disappears when the average density is reduced, and so on).

In the second part of this paper, we address the origin of the inhomogeneity and the possibility that it arises from an electronic phase separation, as indicated by experimental evidences of negative electronic compressibility $[12,13]$. In this framework, we also propose a microscopic mechanism of electronic instability based on the interplay between the electron gas and the ionic countercharges due to ionic-liquid gating.

\section{INHOMOGENEOUS TRANSPORT MODEL}

\section{A. Physical scenario of inhomogeneous 2D crystalline superconductors}

Our work moves from two phenomenological observations: (a) the metal-to-superconductor transition is generically so broad in 2D crystalline superconductors that no sensible fluctuation mechanism can account for it [14-16] [see Figs. 1(a), 1(f), and 1(k)]; (b) when the filling of the 2D electron gas is induced by ionic-liquid gating, the width of the transition is generically broader and it is always accompanied by a tailish character in the low- $T$ part of the resistancevs-temperature curves $R(T)$ [see, e.g., the shaded regions 
in Fig. 1(a)]. This latter feature is much less pronounced in chemically doped systems [17].

To account for these observations, we assume that the electron gas in 2D crystalline superconductors is inhomogeneous, with a metallic matrix hosting puddles that become superconducting below a random local critical temperature $T_{c}$. The length scale of the inhomogeneity is immaterial, provided the puddles are large enough to sustain a superconducting state below the given local $T_{c}$ and the metal-superconductor mixture is fine enough to allow for a good statistical sampling even on small samples a few micrometers large (in oxide interfaces like, e.g., $\mathrm{LaAlO}_{3} / \mathrm{SrTiO}_{3}$, such conditions are met with typical inhomogeneities on the scale of a few hundreds of nanometers [4-7], slightly larger than the superconducting coherence length $\xi \approx 50 \mathrm{~nm}$ ). Before presenting any specific model, we now find it useful to give a pictorial view of the physical situation.

The inhomogeneous metallic state. First of all, we assume that the system has an inhomogeneous density. This density inhomogeneity has minor consequences in the metallic phase because quasiparticles are weakly scattered by extended inhomogeneities. Although a quantum theory for transport in inhomogeneous media is still lacking, the effects of scattering due to extended impurities has already been considered in the past (see, e.g., Ref. [18]). It is known that when the extension of the inhomogeneity is large enough, it hardly affects the mobility of carriers because electrons, when crossing large inhomogeneities at (slightly) different density, are not backscattered (which would strongly degrade currents), rather they are weakly refracted, giving rise to a dominantly forward scattering, which is not detrimental for transport. In other words, metallic states with weakly modulated densities can still display high charge mobility and their transport properties are hardly different from those of clean homogeneous metals. In a phenomenological classical scheme like, e.g., the effective medium theory $[19,20]$, a mixture of metals with slightly different local resistivity cannot be distinguished from a homogeneous metal with the resulting effective resistivity. Owing to the extended nature of disorder in these systems, we also expect that localization effects (usually coming from interference effects in time-reversed electronic paths scattered by quenched pointlike impurities) have minor relevance.

The inhomogeneous superconducting state. The above situation becomes drastically different when some regions start to become superconducting by lowering $T$. The contrast between higher- and lower-density regions is then strongly enhanced because some regions abruptly acquire a vanishing resistance. In this situation, superconducting puddles at higher electron density are embedded in a metallic matrix with large effect on the overall resistivity and other transport properties. In this regard, we notice that several mechanisms can trigger a local superconductivity with even a small local increase of the electron density. In $\mathrm{LaAlO}_{3} / \mathrm{SrTiO}_{3}$ interfaces, for instance, it is known that a superconducting dome arises in the $2 \mathrm{D}$ electron gas when the filling is increased and the Fermi energy exceeds a threshold $E_{c}$ allowing the occupation of additional bands with higher density of states [21,22]. An alternative mechanism could arise from the interplay with a competing phase as it often occurs near a quantum phase transition, where superconductivity, being favored by quantum fluctu-

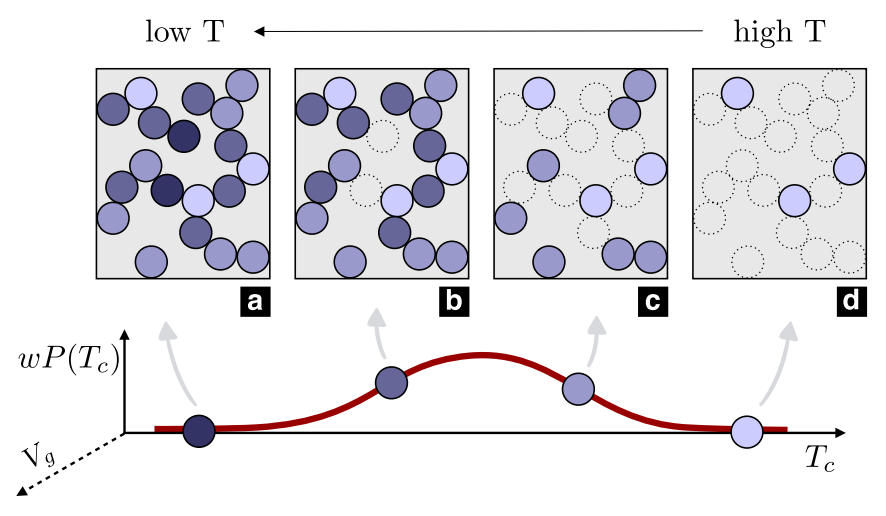

FIG. 2. (Top) Schematic representation of an inhomogeneous 2D crystalline superconductor. At high temperature the metal is weakly inhomogeneous. As the temperature is reduced, from right to left, more and more superconducting puddles (the light-to-dark blue circles) are found with a critical temperature $T_{c}>T$, as highlighted by increasingly darker hues of color. Correspondingly, an increasing fraction of the system becomes superconducting. A zero resistance state is reached as soon as a percolating path is formed. (Bottom) The number of superconducting puddles is ruled by the probability distribution $P\left(T_{c}\right)$ of the critical temperatures and $w$ is the overall fraction of potentially superconducting regions. The distribution and fraction may depend on control parameters like, e.g., the gate voltage $V_{g}$ tuning the electron density of the system.

ations and by the weakening of the competing order, forms a superconducting dome in the phase diagram region around the quantum critical point. These arguments should justify our assumption that even a rather weak inhomogeneity in electron density may result at low temperature into an inhomogeneous mixture of normal and superconducting regions [23].

The distribution of local critical temperatures. We now discuss the corresponding distribution of local critical temperatures. In the context of $\mathrm{LaAlO}_{3} / \mathrm{SrTiO}_{3}$ interface, a simple calculation within BCS theory [6] shows that a rapid increase of the local $T_{c}$ takes place when the chemical potential passes the energy threshold for filling the bands relevant for superconductivity $T_{c} \propto \sqrt{\mu-E_{c}}$. Therefore, weak density variations induce sizable variations on $T_{c}$. In this framework, one can also generically notice that the global $T_{c}$ can vary rapidly with small variations of the average electron density (see, e.g., Fig. 7 of Ref. [2], where the phase diagrams of $\mathrm{ZrNCl}$ and $\mathrm{MoS}_{2}$ are reported). These arguments suggest that mild spatial variation of the density may induce a rather broad distribution of local $T_{c}$ 's.

Although disorder is not a main factor in highly crystalline films, it can still have some effects on the local $T_{c}$ 's: It is known that $T_{c}$ can be substantially reduced by disorder [24] and it is conceivable that fluctuations in the spatial distribution of impurities and/or of the local chemical potential (due to the density inhomogeneity) at the nanoscale induce variations of $T_{c}$ in the superconducting puddles. This provides an additional argument to support the idea that the superconducting puddles may have a rather large distribution $P\left(T_{c}\right)$ of local superconducting critical temperatures. As schematically depicted in Fig. 2, the resistance of the system keeps varying (and possibly vanishes if a percolating cluster of superconducting puddles is formed) when $T$ is progressively decreased around 
the metal-to-superconductor transition. The width of the $T_{c}$ distribution is then directly related to the width $\sigma$ of the average metal-to-superconductor (or high- $T$-to-low- $T$ metal) transition.

In summary, the scenario we consider is characterized by the following: (a) a weak density inhomogeneity that is compatible with a high-mobility metallic state; (b) a mixed metal-superconductor state with a broad transition ruled by the width of the $T_{c}$ distribution of the superconducting puddles; (c) the possible occurrence of a low- $T$ metallic state as a mixture of normal and superconducting regions in the absence of a percolating superconducting cluster: as soon as all the potentially superconducting regions have zero resistance, the overall resistance no longer varies by further decreasing $T$. Due to the highly crystalline character, other mechanisms, like, e.g., Anderson localization, are still not effective at the lowest experimentally accessed temperatures and the resistance appears to saturate to a constant value. In this way, the mysterious low- $T$ quantum metal state acquires a quite natural and simple interpretation: it is just the metallic state of a very clean system in the presence of embedded, nonpercolating superconducting puddles. Other specific features, like, e.g., the tailish $R(T)$, can also be reproduced, as they result from specific structural features of the inhomogeneous clusters. The study of these features of course requires a specific model, that we introduce in the next subsection.

\section{B. Random-resistor network model}

To describe the above physical scenario, we represent the system by a random-resistor network (RRN). We have to capture somewhat opposite characteristics of the data. On the one hand, the resistance curves tend to vanish (or to saturate at a finite value, if percolation does not occur) with a rather long tail, which is the hallmark of a weak long-distance connectivity of the superconducting cluster [5,6,25]. This is because in a (nearly) one-dimensional conductor, a zeroresistance superconducting state is only achieved when all the bonds have become superconducting. This requires a more stringent condition that is realized at a lower temperature than the average $T_{c}$ of the random critical temperature distribution: until the very last resistive bonds are switched off, $R$ stays finite giving rise to the low- $T$ tailish shape of $R(T)$.

On the other hand, when lowering the temperature, already well above the transition, there is a marked decrease of the resistance, which extends over a broad temperature range. This indicates that a whole substantial part of the system is becoming superconducting. To capture this multifaceted physics, we should therefore account for three distinct features, namely, the weak long-distance connectivity of the superconducting network, its bulky character, and the randomness of the critical temperature of its constituents. To this purpose, we conceived the following composite spatially correlated structure (for details, see Appendix):

(1) First of all, we discretize the 2D inhomogeneous metallic gas by taking a 2D square lattice where each resistor is located on the $i$ th bond of the grid [Fig. 3(a)] and can either stay metallic with a typical resistance $R_{0}$ down to the lowest accessible temperature, or become superconducting (i.e., its resistance vanishes) below a given local critical temperature (a)
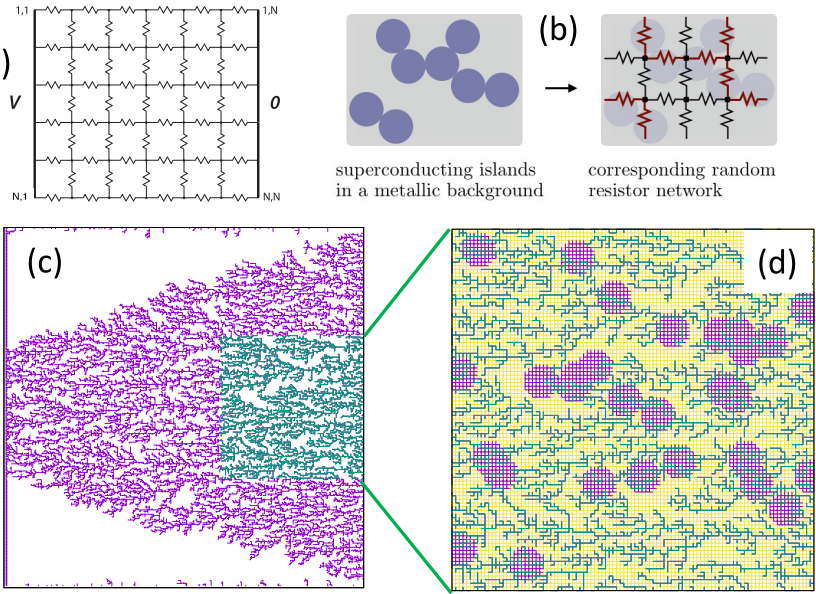

FIG. 3. (a) Schematic view of an $N \times N$ resistor network. The left and right vertical edges are kept at potential $V$ and 0 , respectively. (b) Scheme of the mapping of a system of superconducting islands embedded in a metallic background onto a random-resistor network. The right (b) panel is an enlargement of the 2D square lattice of metallic bonds: the resistors highlighted in red are those that become superconducting below a random local critical temperature $T_{c}$. (c) Example of a filamentary structure produced by letting 50000 particles diffuse across a $250 \times 250$ square lattice, according to the DLA prescription explained in the Appendix. Notice that the underlying 2D square-lattice grid is not reported for clarity (this is the overall blank part of the figure) and only its DLA fractal subset is represented. From this larger fractal, a restricted $100 \times 100$ square sublattice is extracted from the original $250 \times 250$ to serve as a filamentary skeleton for the superconducting component of our RRN. (d) Example of a superconducting cluster of our RRN, obtained superimposing bulkier circular superpuddles (in purple color), of diameter equal to 10 bonds, to the (green) fractal skeleton generated by means of the DLA prescription. In this panel, the underlying $2 \mathrm{D}$ square-lattice grid is reproduced in light yellow.

$T_{c}(i)$, randomly extracted from a probability distribution. Figure 3(b), right panel, displays an enlarged view of the whole 2D square lattice of resistors. The black resistors represent the metallic matrix and keep their resistance $R_{0}$ finite down to the lowest temperature, while the red resistors belong to the potentially superconducting clusters and become superconducting below their local $T_{c}(i)$.

It turns out that the specific shape of the $R(T)$ curves can only be described by a spatially correlated cluster [5,25], and luckily its features are quite informative about this spatial structure.

(2) To distribute spatially these potentially superconducting bonds [the red resistors of Fig. 3(b), right panel], we generate a fractal-like structure using a diffusion limited aggregation (DLA) algorithm and we select a region of the fractal cluster filling our numerical cluster [the green subset in Fig. 3(c)]. This fractal character by no means implies that real systems have a self-similar spatial distribution of superconducting regions, but it is a mere technical tool to generate a spatially correlated cluster with a random and filamentary structure. On top of this faint filamentary skeleton, circular (i.e., bulkier) large puddles (we nickname them superpuddles) are randomly added [the purple circular regions in Fig. 3(d)]: 


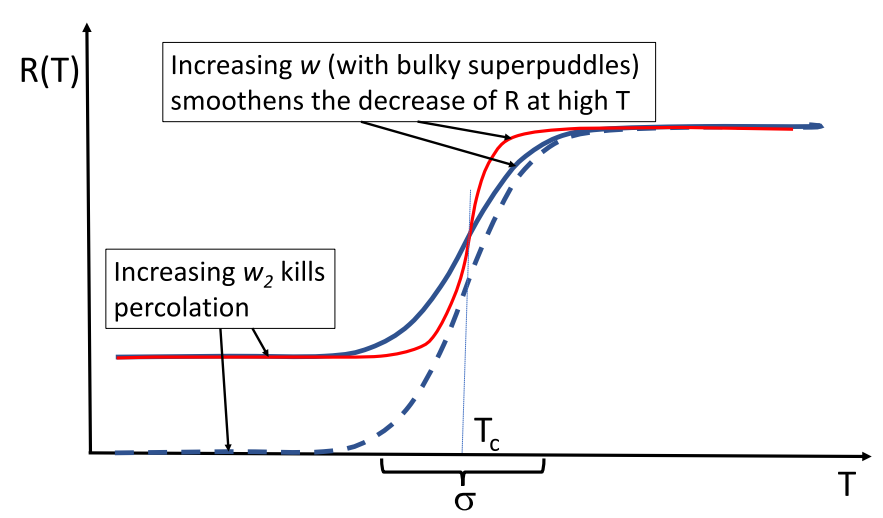

FIG. 4. Schematic view of how the shape of $R(T)$ depends on the various parameters of the model. Increasing $w_{2}$ while keeping $w$ fixed, more superconducting filaments are broken and percolation is lost: the dashed blue line becomes the solid blue line. Increasing $w$ by increasing the number of superpuddles renders more gradual the decrease of $R(T)$ (red-to-blue solid lines). $\bar{T}_{c}$ is the center of the transition, while $\sigma$ rules its width.

We randomly select a superconducting bond of the 2D grid, we attribute it to the superconducting cluster, and we manually add more superconducting bonds in its vicinity, to form a circular patch of superconducting bonds. All the bonds (i.e., the resistors) in the superpuddle are set to have the same random local $T_{c}$. The addition of superpuddles stops when a total weight $w$ is reached to attain a given superconducting fraction in the system (this is one of our fitting parameters). Figures 3(d) and 5(c) display typical structures of the RRN inhomogeneous cluster, where the filamentary skeleton coexists with the circular superpuddles.

When the superconducting cluster does not percolate, the resistance curve $R(T)$ at low $T$ saturates at a finite value. This occurs because the total superconducting weight in the system is low and some breaks occur in the fractal filaments spoiling the connectivity of the superconducting cluster. To describe this situation, we randomly chose some resistors in the filamentary fractal subset of the 2D square-lattice grid and turn them back to metallic [i.e., we manually set their local $T_{c}(i)=0$ ]. The fraction $w_{2}$ of these broken bonds is also adjusted by the fit.

(3) Once the above complex structure of the superconducting cluster is generated, the model is completed by assigning a probability distribution of the random local critical temperatures. For the sake of definiteness, we adopt a Gaussian distribution

$$
P\left(T_{c}\right)=\frac{1}{\sqrt{2 \pi} \sigma} e^{-\frac{\left(T_{c}-\bar{T}_{c}\right)^{2}}{2 \sigma^{2}}}
$$

In summary, our RRN model is characterized by four parameters $\left(\bar{T}_{c}, \sigma, w, w_{2}\right)$, each having its specific effect on the resistance curve and therefore providing information on the structure of the superconducting clusters. Specifically, as schematically described in Fig. $4, \bar{T}_{c}$ and $\sigma$ rule the position and the width of the resistance decrease around the metalto-superconductor (or high- $T$-to-low- $T$ metal) transition. $w$ is the fraction of superconducting regions and, being mostly arranged by the number and size of the superpuddles, it determines the smoothness and gradualness of the decrease of $R(T)$ on the high- $T$ side of the curve [26]. We also notice that the radius of the superpuddles is not a relevant parameter as long as it is not too large in comparison with the size of the RRN [if this is not the case, only a few superpuddles are contained in the cluster and steps appear in $R(T)$ whenever a single superpuddle becomes superconducting]. Finally, $w_{2}$ is the most effective parameter in ruling the long-distance connectivity of the superconducting clusters.

We emphasize that both the bulky superpuddles and the filamentary connections are crucial to reproduce the shape of $R(T)$ : the former give a smooth substantial decrease starting at high temperature, while the latter are responsible for the tailish shape at low temperatures.

\section{Using the RRN to fit the resistance data of 2D superconductors}

Once the random structure of the inhomogeneous RRN is set, we then devote a systematic numerical effort to solve the Ohm's and Kirchhoff's equations on bonds and nodes of the clusters (of typical size $200 \times 200$, see Appendix), thereby determining the local and global currents and voltages, hence the global resistance of the system, to fit the experimental curves. While the results of the fits are given in Figs. 1(a), 1(f), and 1(k) for $\mathrm{ZrNCl}, \mathrm{MoS}_{2}$, and $\mathrm{TiSe}_{2}$ respectively, the corresponding values of the parameters characterizing the cluster geometry $\left(w\right.$ and $\left.w_{2}\right)$ and the $T_{c}(i)$ distribution $\left(\bar{T}_{c}\right.$ and $\sigma$ ) are reported in the lower panels of Fig. 1.

Clearly, our model captures the tailish character of the $R(T)$ curves near the zero-resistance limit, as well as the saturation to finite values when percolation is not achieved. Both these effects are a consequence of the poor long-distance connectivity of our superconducting cluster. In the case of saturation to finite values, the system does not become superconducting even when all resistors inside the subset are switched off because of the lack of a fully percolative superconducting cluster. Therefore, within this percolative scheme, the residual finite resistance remaining at low temperature in some lowdensity samples has a very natural interpretation: it is due to the pristine metallic matrix embedding the (nonpercolating) superconducting puddles.

The model also allows to distinguish two physically different situations: in one case, superconductivity disappears at low $T$ because upon reducing the average electron density the superconducting puddles become less dense and more sparse, while in the second case the disappearance of superconductivity is driven predominantly by a reduction of the average superconducting critical temperature in the puddles, e.g., because of some competing mechanism. Both these situations are found to occur and are reported in Fig. 1. In particular, one can see that in $\mathrm{ZrNCl}$ [Fig. 1(a)] the average local critical temperature only varies from $14 \mathrm{~K}$ at high density to $10 \mathrm{~K}$ at the lowest density [Fig. 1(b)]. Notice that in this case the superconducting fraction [Fig. 1(d)] is so low that the puddles do not percolate and the system stays metallic down to the lowest temperatures. Therefore, the system fails to reach the zeroresistance state even though a substantial part of it is locally superconducting with a rather large $T_{c} \approx 10 \mathrm{~K}$ [Fig. 1(b)]. The moderate reduction of the average $T_{c}$ [Fig. 1(b)] can be 


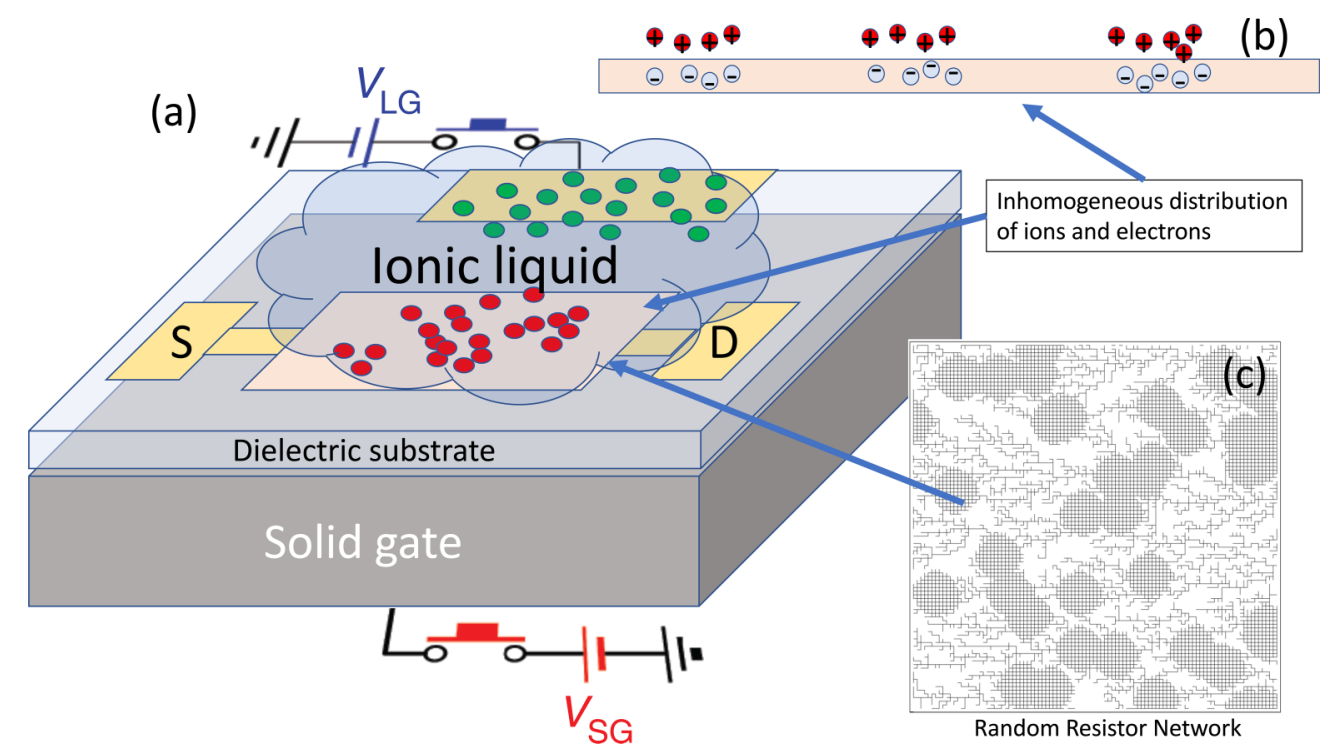

FIG. 5. (a) Schematic representation of a $2 \mathrm{D}$ crystalline superconductors (pink rectangle) on a dielectric substrate in the presence of a metallic back gate (gray region) and a ionic liquid (light-blue droplet). The positive (red) and negative (green) ions are also represented together with the ionic-liquid gating (light orange rectangle). S and D are the source and drain electrodes. (b) Schematic profile of the inhomogeneously doped layer of a 2D crystalline superconductor. (c) $100 \times 100$ cluster of RRN showing the filamentary structure together with the bulkier superpuddles.

explained because the global average reduction of electron density reflects in a reduction of the local density even in the superconducting puddles, thereby inducing a relatively larger effect of quenched impurities. The RRN model even allows to distinguish whether the density reduction affects more the superpuddles [see Figs. 3(d) and 5(c)] or the connecting superconducting filaments. One can see that the high- $T$ parts of the resistance curves at the transition are quite similar, indicating that the bulky part of the superconducting regions is more or less unaffected, while the density decrease affects more the connecting filaments, that disappear with a fraction $w_{2}$, which increases while reducing the gating [Fig. 1(e)]. Of course, some more rapid downward bending of the black curve at $V_{l g}=5.0 \mathrm{~V}$ than in the $V_{l g}=5.5,6.5 \mathrm{~V}$ cases indicates that the density decrease also reduces the weight of the superpuddles, but this is comparatively less relevant for transport. In other words, we see that for $\mathrm{ZrNCl}$ percolation is destroyed because the underlying geometrical support is strongly affected (namely, many connecting bonds no longer superconduct, see the increase of $w_{2}$ by reducing the overall density), while the distribution of local $T_{c}$ 's is modified in a comparatively less relevant manner.

On the other hand, transition metal dichalcogenides are notoriously characterized by a strong tendency to form chargedensity waves (CDWs), which compete with superconductivity. Although it is overwhelmingly difficult to microscopically describe this interplay and competition, it is clear that reducing the average density in these systems strengthens the CDW order, which has a strong influence also inside the superconducting puddles. Therefore, the local $T_{c}$ 's are suppressed and vanish at low enough electron density. In this case, the disappearance of the superconducting phase does not occur because of a lack of percolation, but because the weakest part of the superconducting subset loses its superconducting character. This can be easily recognized in the lack of a saturating low-T resistance in Figs. 1(f) and 1(k) for $\mathrm{MoS}_{2}$ and $\mathrm{TiSe}_{2}$. In this case, therefore, contrary to the $\mathrm{ZrNCl}$ case, the geometrical support of superconductivity is less affected, while the local $T_{c}$ 's are generically reduced by the competition with CDW and their overall distribution is shifted to lower values.

\section{MECHANISMS OF ELECTRONIC PHASE SEPARATION}

Once the inhomogeneous character of the ionic-liquid gated 2D crystalline superconductors is assessed via the above phenomenological analysis, the crucial question is left about the origin of this inhomogeneity. Due to the quite general occurrence of such inhomogeneity in different systems doped by ionic-liquid gating, we propose here that the $2 \mathrm{D}$ electron gas in these systems may become thermodynamically unstable and undergo an electronic phase separation, thereby displaying a negative compressibility due to the combined action of the confining potential well and of the gating ionic countercharges. Of course, as it will be discussed in the last part of this paper, several mechanisms intervene to stop the full development of the electronic phase separation, giving rise to a final thermodynamically stable (but inhomogeneous) system. Here, we only focus on the electronic part of the system and its interplay with the ions of the liquid gate to explain the source of instability. We describe the 2D electron gas as a free-electron gas confined in a potential well that quantizes the electron motion in the direction perpendicular to the interface. The depth of the well depends on the amount of countercharges (i.e., the positive ions in the liquid nearby the $2 \mathrm{D}$ crystalline superconductor) per $2 \mathrm{D}$ unit cell $v$, so that the band dispersion of the 2D electron gas will be henceforth written as $\varepsilon_{k}=\varepsilon_{0}(v)+\frac{k^{2}}{2 m}$, where $\varepsilon_{0}(v)$ is the quantized level in the confining potential well, above which the $2 \mathrm{D}$ band 
dispersion arises, $m$ is a suitable effective mass, and $k$ is the $2 \mathrm{D}$ quasimomentum parallel to the interface. For a given density of countercharges $v$, the grand-canonical electronic Hamiltonian reads as

$$
\mathcal{H}=\sum_{k, \sigma}\left(\varepsilon_{k}-\mu\right) \hat{n}_{k, \sigma}-\lambda\left(\sum_{k, \sigma} \hat{n}_{k, \sigma}-N v\right),
$$

where $\mu$ is the electron chemical potential, $N$ is the number of cells, and the Lagrange multiplier $\lambda$ enforces the constraint that each electron comes from a dopant countercharge, i.e., the average electron density $n=v . \hat{n}_{k, \sigma}$ is the operator that counts the number of electrons of quasimomentum $k$ and $\operatorname{spin} \sigma$.

We assign a bandwidth $W$ to the $2 \mathrm{D}$ electron gas, and write the DOS as $N_{0}=1 / W$. Then, the condition of overall neutrality at $T=0$ becomes

$$
n=\frac{2}{W} \int_{\varepsilon_{0}(v)}^{\mu+\lambda} d \varepsilon=v
$$

since the Fermi statistics counts the states with negative eigenvalues of $\mathcal{H}, \varepsilon_{k}-\mu-\lambda<0$, the minimum value of $\varepsilon_{k}$ being $\varepsilon_{0}(v)$. The factor of 2 accounts for the spin multiplicity. Then, the $T=0$ grand-canonical thermodynamical potential per unit cell reads as

$$
\begin{aligned}
\omega & =\frac{2}{W} \int_{\varepsilon_{0}(v)}^{\mu+\lambda}(\varepsilon-\mu-\lambda) d \varepsilon+\lambda \nu+\omega_{0}(\nu) \\
& =-\frac{1}{W}\left[\mu+\lambda-\varepsilon_{0}(\nu)\right]^{2}+\lambda \nu+\omega_{0}(v),
\end{aligned}
$$

where $\omega_{0}(v)=\frac{1}{2} A v^{2}+\frac{1}{4} B v^{4}$ is the countercharge contribution, the first term resulting from the countercharge inverse compressibility, and the second term modeling the cost of increasing the countercharge density and stabilizing the system against large variations of $v$, with $A$ and $B$ suitable constants. It is worth noticing that $A$ arises from the short-range part of the ion-ion interaction only because the Coulomb long-range part is exactly compensated by the electrons $(n=v)$. This short-range character and the absence of kinetic energy for the ions renders this term practically negligible (at least in comparison with the much larger inverse electron compressibility $\sim W$ ). Quite relevant (but hard to estimate from first principles) is the $B$ term acting when the ion density increases and stabilizing the ion system against a high-density collapse.

Starting from Eq. (4), the average number of electrons per unit cell can be obtained as $n=-\partial_{\mu} \omega=$ $2 W^{-1}\left[\mu+\lambda-\varepsilon_{0}(v)\right]$, which is the result of the integration of Eq. (3) and yields $\mu=\frac{1}{2} W n-\lambda+\varepsilon_{0}(v)$. The condition

$$
\partial_{\lambda} \omega=v-\frac{2}{W}\left[\mu+\lambda-\varepsilon_{0}(v)\right]=0
$$

enforces the constraint $n=v$. Finally, imposing equilibrium with respect to $v$ gives

$$
\partial_{\nu} \omega=\frac{2}{W}\left[\mu+\lambda-\varepsilon_{0}(\nu)\right] \partial_{\nu} \varepsilon_{0}+\lambda+\partial_{\nu} \omega_{0}=0,
$$

hence $\lambda=-\left(n \partial_{\nu} \varepsilon_{0}+\partial_{\nu} \omega_{0}\right)$.

Within an electrostatic continuous model it can easily be shown that the depth of the confining potential well increases
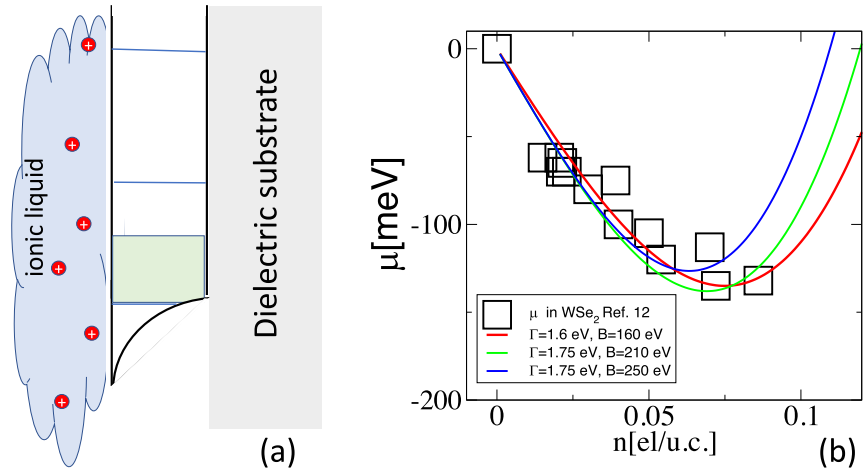

FIG. 6. (a) Schematic representation of the electronic potential well confining the 2D electron gas (green area) between the dielectric substrate (gray area) and the ionic liquid (light blue area). (b) Chemical potential vs electron density in the $2 \mathrm{D}$ electron gas in the film. The parameters are $A=0, \Gamma=1.75 \mathrm{eV}, B=210 \mathrm{eV}$ (green curve), and $B=250 \mathrm{eV}$ (blue curve) and $\Gamma=1.6 \mathrm{eV}, B=160 \mathrm{eV}$ (red curve). The squares are data from Ref. [13] obtained from ARPES experiments on surface-doped $\mathrm{WSe}_{2}$.

linearly with $v$ : the more abundant are the positive ions in the liquid gate side and the lower is the electrostatic confining energy trapping the electron gas at the surface. On the other hand, trapped charges may counteract this dependence when $v$ is small: A small density of liquid ions induces a corresponding small density of electrons, which, in this poorly screened situation, are trapped as bound states around the positively charged liquid ions. In this situation, the electronic level $\varepsilon_{0}(v)$ is locked to the (random) bound-state levels. To describe this low-density situation and its smooth evolution to the intermediate density situation, in which $\varepsilon_{0}(v)$ linearly decreases because of the electrostatic interaction with the charged ions, we phenomenologically write $\varepsilon_{0}(v)=-\Gamma v^{2} /\left(v+v_{0}\right)$, where $\nu_{0}$ is the threshold value above which a linear dependence is recovered and $\Gamma$ is a constant. The parameter $\Gamma$, embodying the dependence of the bottom of the 2D electron band on the ion density, can be expressed in terms of the capacitance of the interface $\tilde{C}$, according to the relation $\Gamma=|e| /\left(2 \tilde{C} a^{2}\right)$, where $e$ is the electron charge and $a$ is the lattice spacing of the $2 \mathrm{D}$ unit cell. Typical numbers are $\tilde{C} \approx 10 \mu \mathrm{F} \mathrm{cm}^{-2}$ and $a \approx 3 \times 10^{-8} \mathrm{~cm}$ [27], yielding $\Gamma \approx 10 \mathrm{eV}$. A more accurate (self-consistent) treatment of the potential well confining the electrons at the interface, which is beyond the scope of this work, could provide a better estimate of the numerical prefactor relating $\Gamma$ to the typical interfacial potential scale $|e| /\left(\tilde{C} a^{2}\right)$. The typical electron bandwidth can be estimated as $W \approx 1 \mathrm{eV}$ [28]. Putting together all the pieces, we can now write the electron chemical potential as

$$
\mu=\frac{W}{2} n+n\left(A+B n^{2}\right)-\Gamma \frac{n^{2}\left(2 n+3 v_{0}\right)}{\left(n+v_{0}\right)^{2}} .
$$

As it is readily seen, when $v_{0}=0$, and for $\frac{W}{2}+A-2 \Gamma<0$, the inverse compressibility $\kappa^{-1}=\partial_{n} \mu$ is negative at small $n$, so that the system is unstable against electronic phase separation, as shown in Fig. 6 for a typical parameter set that compares rather well with the negative compressibility observed in photoemission experiments in surface-doped $\mathrm{WSe}_{2}$. 


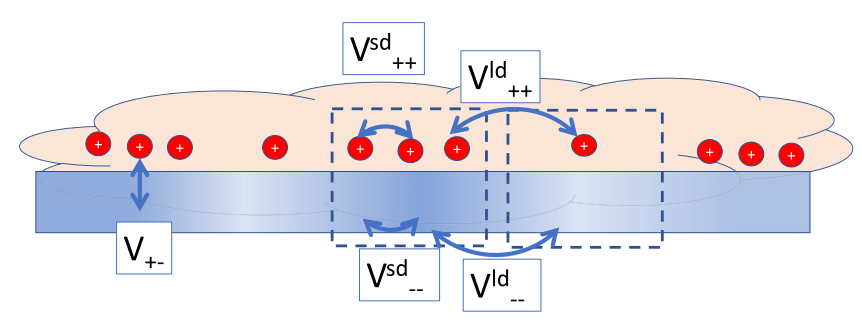

FIG. 7. Schematic representation of the inhomogeneous electronic distribution (blue and light blue regions) accompanied by the inhomogeneous ionic distribution (red circles) embedded in the liquid droplet.

We point out that, if electronic phase separation is allowed to fully develop, the access to the negative compressibility region is forbidden, and the customary Maxwell construction must be used to determine the inhomogeneous composition of the phase-separated system. The fact that a negative electron compressibility is experimentally measured in surface-doped $\mathrm{WSe}_{2}$ [13] may indicate that chemical dopants are not as mobile as ions in the liquid, thereby leaving the possibility that electronic phase separation is frustrated by residual longrange Coulomb forces, due to the lack of perfect compensation between electrons and countercharges. In this case, the short-range electron compressibility could stay negative, while the electron charge density is spatially distributed to find a compromise between the local tendency to electronic phase separation and the Coulombic cost of a charge-unbalanced density profile [29]. A somewhat similar situation occurs in the model of Ref. [30], which may be relevant at low electron density, or in badly metallic systems.

A finite $v_{0}$ would stabilize the compressibility at small $n$, while the term proportional to $B$ always stabilizes the system against large variations of the density, yielding a finite density window where the system is unstable, and separates into two metallic phases, with higher and lower electron density. For $v_{0}=0$, the low-density phase is (band) insulating, while a finite $v_{0}$ may allow for a low-density metallic phase to occur.

\section{DISCUSSION}

Aside from its formal implementation, the above model is a rather general representation of the physical situation schematically depicted in Fig. 7. The ion-ion repulsion is strongest at short distances $(s d), V_{++}^{s d}$, preventing excessive ion accumulation in small volumes, and within our phenomenological coarse-grained model this effect is modeled by the rapid increase of the quartic term in the free energy [ $B$ term in $\left.\omega_{0}(v)\right]$. At large distances $(l d)$, despite the poor screening inside the liquid droplet, the underlying electrons balance the ionic charge and give rise to a small (dipolarlike) repulsion $V_{++}^{l d}$ (at larger scales, the inhomogeneous regions, represented by the dashed contours in Fig. 7, are essentially neutral). The same holds for the electron-electron repulsion, which is weak at large distances $V_{--}^{l d}$, due to compensating ionic countercharges and may be larger at short distances $V_{--}^{s d}$. For electrons there is the additional effect of mutual screening and high charge compressibility (large DOS of the 2D electron gas metallic state). In this scheme, a large attractive contribution is present between the mobile electrons and the mobile (nearly frozen) ions at high (low) temperature. This electrostatic gain is such that the system may find it convenient to have moderately higher ionic and electron densities to exploit this attraction. Of course, if the density fluctuation becomes large, the repulsive cost (i.e., the $B$ term of the ion-ion free energy) stops the aggregation tendency. Therefore, aside from the high screening inside the metallic layer, a negative electronic compressibility is favored by the reciprocal charge compensation. Formally, this is represented by the constraint $n=v$.

On a general basis, we observe that an inhomogeneous state may result from rather generic attractive interactions induced by the interplay between the confined 2D electron gas and the countercharges coming from the gate, from chemical doping, or oxygen vacancies, as recently proposed for the oxide heterostructures [11,31]. The inversion-asymmetric electric field confining the 2D electron gas may also induce a strong Rashba spin-orbit coupling, which depends on the local electron density and may provide an additional source of effective electronic attraction [10,32]. These effective attractions can produce an electronic phase separation in the $2 \mathrm{D}$ electron gas as it is also supported by experimental evidences of a negative electronic compressibility in graphene- $\mathrm{MoS}_{2}$ heterostructures [12], in $\mathrm{WS}_{2}$ [13], in $\mathrm{SrTiO}_{3}$ surface [33], and in $\mathrm{LaAlO}_{3} / \mathrm{SrTiO}_{3}$ interfaces [34]. Of course, specific features are present in each system that render the electronic phase separation specific. For instance, the size of the inhomogeneous regions depends on the frustrating effects of electron-electron and countercharge-countercharge Coulomb repulsions, which in turn depend on their mobility and on the screening in the various parts of the system. In the present case, we have considered a model where the charges of ionic-liquid gating favor an extended electronic phase separation thanks to their high mobility (above their freezing temperature) that allows a large-scale segregation of electrons and ions while keeping an overall quasineutrality. We notice that our proposed mechanism may cooperate with electron inhomogeneity induced by the frozen ionic liquid locally detaching from the sample surface [35]. Of course, when electrons are introduced by chemical doping, the countercharges are much less mobile and a more even distribution of inhomogeneity (if any) is expected. This is why in this case the metal-to-superconductor transition is generically narrower and no tail is present in the $R(T)$ curves [see, e.g., Figs. 6(c) and 6(e) in Ref. [17]]. Notice, however, that at low doping [Fig. 6(a) in Ref. [17]] relative fluctuations in the distribution of the dopants become more important and again a broad transition is found. The situation may be even more intricate when competing phases like CDWs are present, as in the case of the domain-wall formation recently discovered by scanning tunneling experiments [36] in Cu-intercalated $1 \mathrm{~T}-\mathrm{TiSe}_{2}$. Still, it is worth noticing that also in this case our phenomenological RRN model can capture and describe the rather filamentary structure of the metallic domain walls responsible for incommensuration in the CDW and superconductivity in this system. We also notice that a suitable choice of the temperature dependence of the resistance in the normal metallic regions also allows a very good description of the onset of superconductivity in $\mathrm{MoS}_{2}$ on amorphous substrates [37]. 
Despite the variety of situations and of microscopic mechanisms giving rise to the inhomogeneity, our phenomenological RRN model is quite general as long as the superconducting regions are large enough to sustain a local superconducting state. The physics of the system, at a mesoscopic level, is fully encoded by the spatial distribution of the cluster, its connectivity, and the parameters of the $T_{c}$ distribution. This is why upon reducing the average electron density, the model also allows to distinguish and describe the physically very different situations reported in Figs. 1(a), 1(f), and 1(k), where superconductivity disappears because the puddles become sparse and less dense or because the local $T_{c}$ is degraded by the competition with CDWs. When the spatial distribution is filamentary, the low connectivity of the RRN accounts for the tailish shape of $R(T)$, with few nonsuperconducting puddles preventing the zero-resistance state and $R(T)$ staying finite until the very last puddles become superconducting at low $T$. On the contrary, when the puddles are more evenly distributed, the connectivity is large, percolating paths are easier to find, and $R(T)$ vanishes without the long tail.

Our inhomogeneous scenario can be tested by critical current experiments: despite the complex structure of the superconducting cluster, in the proximity of the critical current, transport should be ruled by the weakest links coupling nearby puddles, so that the critical current and its temperature and magnetic field dependencies are expected to be well described by the behavior of a single (or a few) Josephson junction(s). This behavior has already been found in $\mathrm{LaAlO}_{3} / \mathrm{SrTiO}_{3}$ interfaces [7]. In the same systems, a loosely connected (filamentary) regime has also been identified in radio-frequency measurements of the dynamical conductivity to obtain the superfluid density [38]. We suggest that similar experiments in 2D crystalline superconducting systems would provide valuable information.

\section{ACKNOWLEDGMENTS}

We acknowledge stimulating discussions with L. Benfatto, C. Castellani, and F. Mauri and valuable correspondence with Y. Saito and B. Shklovskii. This work has been supported by the Sapienza Università di Roma with Project No. RM11715C642E8370.

\section{APPENDIX: CONSTRUCTION OF THE RANDOM SUPERCONDUCTING CLUSTER}

We give here some details on the construction of the RRN modeling the inhomogeneous $2 \mathrm{D}$ crystalline superconducting systems discussed in Sec. II.

(i) We start from a $2 \mathrm{D}$ array of resistors meeting at the nodes of a 2D $N \times N$ lattice [see Fig. 3(a)]. The two extremal columns ( 1 and $N$ ) of this array represent the external leads at potential $V$ and 0 , respectively. Each bond $(i, j, \hat{x})$, along $x$, or $(i, j, \hat{y})$, along $y$, corresponds to a resistor. Each resistor represents a metallic or superconducting region connected to six neighboring regions as shown in the right-hand side of Fig. 3(b). A bond $(i, j, \hat{e})$ is completely characterized by the current $I(i, j, \hat{e})$ flowing through it and by its resistance $R(i, j, \hat{e})$. The relation between the local resistance $R(i, j, \hat{e})$, the local critical temperature $T_{c}(i, j, \hat{e})$, and the temperature
$T$ follows from the nature of the system intended to study. Here, the form of the relation $R(i, j, \hat{e})=R\left[T ; T_{c}(i, j, \hat{e})\right]$ depends on whether the effects of disorder should be introduced through a distribution of the critical temperatures and/or through a specific dependence of the local resistance on the (critical) temperature. Given that we will be mainly interested in the effects of the distribution of critical temperatures and of spatial correlations between the islands, we discard the latter possibility in order to avoid blurring these effects through complicated $T$ and $T_{c}$ dependencies. We thus consider the simplest case of a binary resistor:

$$
\begin{gathered}
R(i, j, \hat{e})=R_{0}: T>T_{c}(i, j, \hat{e}), \\
0: T<T_{c}(i, j, \hat{e}) .
\end{gathered}
$$

This assumption is reasonable whenever the temperature range in which the resistance of a single resistor goes from its normal-state value $R_{0}$ to 0 is much smaller than the width of the $T_{c}$ distribution [25]. In addition, the normal-state resistivity of each superconducting bond is independent of its critical temperature.

(ii) The second step consists in forming the regions of the $2 \mathrm{D}$ crystalline superconductor, which at low enough temperature become spatially correlated superconducting regions. It turns out that to fit the peculiar shape of the resistance curves of Fig. 1, two geometric ingredients are needed. On the one hand, bulky regions must become superconducting to account for the smooth marked decrease of the resistance from the high-temperature side of the metal-to-superconductor transition. On the other hand, the tailish shape of $R(T)$ on the low- $T$ side of the transition can only be reproduced by weakly connected regions. To this purpose, we proceed as follows (see also Sec. II and particularly Fig. 3): (1) we generate a filamentary weakly connected fractal-like structure and then (2) we decorate this random nearly one-dimensional tree with larger $2 \mathrm{D}$ circular puddles. To generate the fractal-like structure we adopt a a simple growth process known as diffusion-limited aggregation (DLA). Its construction is as follows: a particle is released at the left edge of a $2 \mathrm{D}$ lattice and let diffuse to the right. More precisely, the particle moves one bond to the right and then with equal probability one bond up or down. This sequence is iterated until the particle stops, as soon as it reaches the top, bottom, or right edge, where it sticks. Then, other particles are launched one after another and halted either when reaching one of the three edges or a bond already occupied by one of the previously diffused particles. The cluster obtained in a $250 \times 250$ square lattice after diffusing 50000 particles is defined by the bonds where the particles stick. Due to a saturation at the left edge, the total number of superconducting bonds only amounts to about 25000 . Once this large cluster is obtained [see Fig. 3(c)] we select a $100 \times 100$ sublattice as shown in the green part of Fig. 3(c). The restriction is made to yield a more physical case where the low-dimensional cluster covers the whole sample. So, henceforth we consider restricted lattices ranging from $100 \times 100$ to $200 \times 200$ sites, where only bonds belonging to the cluster are assigned a finite critical temperature $T_{c}$. The other bonds belonging to the rest of the 2D square-lattice grid are not reported in Fig. 3(c) for clarity and appear as the white 
background regions. In Fig. 3(d) they are instead reported in light yellow and they form a resistive background with the typical resistivity $R_{0}$ at all temperatures. The filamentary skeleton of the system is then extended by bulkier 2D patches of circular shape with a diameter ranging from 5 to 30 bonds, leading to structures as shown in Fig. 3(d)

(iii) Once the spatially correlated structure of the superconducting subset of the $2 \mathrm{D}$ crystalline superconductor is formed, the random character of superconductivity in this subsystem remains to be implemented. The physical rationale is that, once intrinsic mechanisms induce density inhomogeneities, substantial fluctuations of the local superconducting properties can arise from a density-dependent local $T_{c}$ or from fluctuations of the quenched disorder. The critical $T_{c}$ is the simplest physical property (and phenomenological parameter) that can reflect the randomly distributed superconducting properties. Thus, we considered for the sake of definiteness the Gaussian distribution of $T_{c}$ 's reported in Eq. (1). When the temperature is progressively decreased, the regions with $T_{c}>T$ become superconducting, enlarging the superconducting regions (see Fig. 2) inside the chosen spatially correlated subset, according to the schematic view of Fig. 3(d).

Notice that the additional gate-potential axis is present in Fig. 2: the increased average number of electrons is reflected in the increase of the total fraction $w$ of the superconducting regions $\left[P\left(T_{c}\right)\right.$ being normalized to one].

(iv) With the above steps the resistances are set, forming the desired geometrical structure of a mixture of filamentary and large puddles (superpuddles), equipped with a randomly chosen local critical temperature. A fixed voltage difference $V$ is applied between the two vertical edges of the lattice, while the horizontal edges have open boundary conditions [see Fig. 3(a)]. The physics of the RRN is governed by the usual equations of electrostatics

$$
\mathbf{J}(\mathbf{r})=-\sigma(\mathbf{r}) \nabla V(\mathbf{r}) ; \quad \nabla \cdot \mathbf{J}(\mathbf{r})=0,
$$

where $\mathbf{J}(\mathbf{r})$ is the current density and $\sigma(\mathbf{r})$ is the conductivity at position $\mathbf{r}$. In the discrete case of a square lattice, these equations simplify to Ohm's and Kirchoff's laws

$$
\begin{aligned}
R(i, j, \hat{x}) I(i, j, \hat{x}) & =V(i, j)-V(i, j+1), \\
R(i, j, \hat{y}) I(i, j, \hat{y}) & =V(i, j)-V(i+1, j), \\
\sum_{\langle\hat{e}\rangle} I(i, j, \hat{e}) & =0,
\end{aligned}
$$

where $V(i, j)$ is the electrostatic potential of site $(i, j)$ and the symbol $\langle\hat{e}\rangle$ restricts the sum over all bonds surrounding a given node $(i, j)$. Implementing the above equations for each of the $N^{2}$ nodes and for each of the $2 N^{2}-2 N$ bonds, one obtains a set of $3 N^{2}-2 N$ linear equations, which determines the $N^{2}$ voltages of the nodes and the $2 N^{2}-2 N$ currents of the bonds. In other words, one needs to solve a system of linear equations $\mathbf{A b}=\mathbf{y}$ where the elements of the matrix $\mathbf{A}$ consist of either $\pm 1, \pm R_{0}$, or 0 , the vector $\mathbf{b}$ contains the unknown potentials and currents, and the vector $\mathbf{y}$ contains the known terms which are either $V$ or 0 . Once the system is solved, the total current $I$ flowing from one edge to the other can be calculated by summing the $N$ currents crossing any of the $N$ vertical cross sections of the array

$$
I=\sum_{i=1}^{N} I(i, j, \hat{x}) .
$$

Due to charge conservation, this sum is independent of $j$. The ratio $V / I$ then determines the global resistance of the cluster at a given temperature. As the temperature is decreased, more and more bonds become superconducting and the global resistance decreases. If at a certain temperature $T_{p}$ a connected superconducting path joins the leftmost and the rightmost edges, the resistance of the network drops to zero, i.e., a percolative metal-to-superconductor phase transition occurs.
[1] A. K. Geim and I. V. Grigorieva, Nature (London) 499, 419 (2013).

[2] Y. Saito, T. Nojima, and Y. Iwasa, Nat. Rev. Mater. 2, 16094 (2016).

[3] M. Tinkham, Introduction to Superconductivity, (McGraw-Hill, New York, 1996).

[4] J. Biscaras, N. Bergeal, S. Hurand, C. Feuillet-Palma, A. Rastogi, R. C. Budhani, M. Grilli, S. Caprara, and J. Lesueur, Nat. Mater. 12, 542 (2013).

[5] D. Bucheli, S. Caprara, C. Castellani, and M. Grilli, New J. Phys. 15, 023014 (2013).

[6] S. Caprara, J. Biscaras, N. Bergeal, D. Bucheli, S. Hurand, C. Feuillet-Palma, A. Rastogi, R. C. Budhani, J. Lesueur, and M. Grilli, Phys. Rev. B 88, 020504(R) (2013).

[7] G. E. D. K. Prawiroatmodjo, F. Trier, D. V. Christensen, Y. Chen, N. Pryds, and T. S. Jespersen, Phys. Rev. B 93, 184504 (2016).

[8] S. Shen, Y. Xing, P. Wang, H. Liu, H. Fu, Y. Zhang, L. He, X. C. Xie, X. Lin, J. Nie, and J. Wang, Phys. Rev. B 94, 144517 (2016).
[9] Y. Saito, T. Nojima, and Y. Iwasa, Nat. Commun. 9, 778 (2018).

[10] S. Caprara, F. Peronaci, and M. Grilli, Phys. Rev. Lett. 109, 196401 (2012).

[11] N. Scopigno, D. Bucheli, S. Caprara, J. Biscaras, N. Bergeal, J. Lesueur, and M. Grilli, Phys. Rev. Lett. 116, 026804 (2016).

[12] S. Larentis, J. R. Tolsma, B. Fallahazad, D. C. Dillen, K. Kim, A. H. MacDonald, and E. Tutuc, Nano Lett. 14, 2039 (2014).

[13] J. M. Riley, W. Meevasana, L. Bawden, M. Asakawa, T. Takayama, T. Eknapakul, T. K. Kim, M. Hoesch, S.-K. Mo, H. Takagi, T. Sasagawa, M. S. Bahramy, and P. D. C. King, Nat. Nanotechnol. 10, 1043 (2015).

[14] J. T. Ye, Y. J. Zhang, R. Akashi, M. S. Bahramy, R. Arita, and Y. Iwasa, Science 338, 1193 (2012).

[15] Y. Saito, Y. Kasahara, J. Ye, Y. Iwasa, and T. Nojima, Science 350, 409 (2015).

[16] L. J. Li, E. C. T. O’Farrell, K. P. Loh, G. Eda, B. Özyilmaz, and A. H. C. Neto, Nature (London) 529, 185 (2015).

[17] D. Bhoi, S. Khim, W. Nam, B. S. Lee, Chanhee Kim, B.-G. Jeon, B. H. Min, S. Park, and K. H. Kim, Sci. Rep. 6, 24068 (2016) 
[18] L. Zhu, P. J. Hirschfeld, and D. J. Scalapino, Phys. Rev. B 70, 214503 (2004).

[19] R. Landauer, in Electrical Transport and Optical Properties of Inhomogeneous Media, edited by J. C. Garland and D. B. Tanner (AIP, New York, 1978).

[20] S. Kirkpatrick, Rev. Mod. Phys. 45, 574 (1973).

[21] J. Biscaras, N. Bergeal, S. Hurand, C. Grossetete, A. Rastogi, R. C. Budhani, D. LeBoeuf, C. Proust, and J. Lesueur, Phys. Rev. Lett. 108, 247004 (2012).

[22] A. Joshua, S. Pecker, J. Ruhman, E. Altman, and S. Ilani, Nat. Commun. 3, 1129 (2012).

[23] S. Caprara, N. Bergeal, J. Lesueur, and M. Grilli, J. Phys.: Condens. Matter 27, 425701 (2015).

[24] A. M. Finkelstein, Pis'ma Zh. Eksp. Teor. Fiz. 45, 37 (1987) [Sov. Phys.-JETP Lett. 45, 46 (1987)].

[25] S. Caprara, M. Grilli, L. Benfatto, and C. Castellani, Phys. Rev. B 84, 014514 (2011).

[26] Indeed, by increasing the number of superpuddles the $R(T)$ curve moves downwards in the right side of the transition and has a smaller slope (see Fig. 4). On the contrary, if one were to consider a strictly $1 \mathrm{D}$ wire embedded in a $2 \mathrm{D}$ square in the absence of superpuddles, in the large-system limit, $R(T)$ would stay constant until the whole wire becomes superconducting. Only at this point the resistivity would vanish with a sudden drop to zero. This limiting case illustrates the opposite effect of making the system more 1D-like without the superpuddles.

[27] W. Shi, J. Ye, Y. Zhang, R. Suzuki, M. Yoshida, J. Miyazaki, N. Inoue, Y. Saito, and Y. Iwasa, Sci. Rep. 5, 12534 (2015).
[28] W. S. Yun, S. W. Han, S. C. Hong, I. G. Kim, and J. D. Lee, Phys. Rev. B 85, 033305 (2012).

[29] J. Lorenzana, C. Castellani, and C. Di Castro, Phys. Rev. B 64, 235127 (2001); 64, 235128 (2001); C. Ortix, J. Lorenzana, and C. Di Castro, ibid. 73, 245117 (2006).

[30] B. Skinner and B. I. Shklovskii, Phys. Rev. B 82, 155111 (2010).

[31] N. Bovenzi, F. Finocchiaro, N. Scopigno, D. Bucheli, S. Caprara, G. Seibold, and M. Grilli, J. Supercond. Novel Magn. 28, 1273 (2015).

[32] D. Bucheli, M. Grilli, F. Peronaci, G. Seibold, and S. Caprara, Phys. Rev. B 89, 195448 (2014).

[33] L. Dudy, M. Sing, P. Scheiderer, J. D. Denlinger, P. Schütz, J. Gabel, M. Buchwald, C. Schlueter, T.-L. Lee, and R. Claessen, Adv. Mater. 28, 7443 (2016).

[34] L. Li, C. Richter, S. Paetel, T. Kopp, J. Mannhart, and R. C. Ashoori, Science 332, 825 (2011).

[35] S. Jo, D. Costanzo, H. Berger, and A. F. Morpurgo, Nano Lett. 15, 1197 (2015).

[36] S. Yan, D. Iaia, E. Morosan, E. Fradkin, P. Abbamonte, and V. Madhavan, Phys. Rev. Lett. 118, 106405 (2017).

[37] J. Biscaras, Z. Chen, A. Paradisi, and A. Shukla, Nat. Commun. 6, 8826 (2015).

[38] G. Singh, A. Jouan, L. Benfatto, F. Couedo, P. Kumar, A. Dogra, R. Budhani, S. Caprara, M. Grilli, E. Lesne, A. Barthélémy, M. Bibes, C. Feuillet-Palma, J. Lesueur, and N. Bergeal, Nat. Commun. 9, 407 (2018). 